This item was submitted to Loughborough's Research Repository by the author.

Items in Figshare are protected by copyright, with all rights reserved, unless otherwise indicated.

\title{
Stochastic dynamics of the diffusive Haken model with subthreshold periodic
} forcing

PLEASE CITE THE PUBLISHED VERSION

PUBLISHER

(C) American Physical Society

LICENCE

CC BY-NC-ND 4.0

REPOSITORY RECORD

Bressloff, P.C., and Peter Roper. 2019. "Stochastic Dynamics of the Diffusive Haken Model with Subthreshold Periodic Forcing”. figshare. https://hdl.handle.net/2134/1728. 


\title{
Stochastic dynamics of the diffusive Haken model with subthreshold periodic forcing
}

\author{
Paul C. Bressloff and Peter Roper \\ Nonlinear and Complex Systems Group, Department of Mathematical Sciences, Loughborough University, Loughborough, \\ Leicestershire LE11 3TU, United Kingdom
}

(Received 12 December 1997)

\begin{abstract}
We demonstrate a noisy resonance phenomenon in a winner-takes-all neural network. We derive an expression for the theoretical transition rate between states and show that this rate matches the driving frequency. We further show that this effect persists when a diffusive coupling is introduced into the network leading to a more robust system. [S1063-651X(98)00308-0]
\end{abstract}

PACS number(s): 87.10.+e, 05.40.+j

\section{INTRODUCTION}

Neurobiologists have noted that in many regions of the cortex, groups of adjacent neurons appear to form higher functional units that serve to analyze some particular stimulus feature such as the orientation of an edge of an image [1], or the position of a sensory stimulus on the skin [2]. Neural network models of the formation and behavior of these coherent structures in brain activity generally involve two aspects: (i) a selection mechanism that determines the center of a localized excitation in response to an input, and (ii) an interaction mechanism that serves to spread the response over a neighboring region of the network, leading to a distributed response. Haken [3] has constructed a simple neural network model to dynamically implement the selection mechanism. The network obeys a competitive gradient dynamics and has ground states that are strictly localized states: a single neuron is active and all others quiescent. Recent work [4-6] has extended this model to take into account a simple interaction mechanism, leading to a distributed representation.

Another important subject currently the focus of much attention is that of noise induced phenomena in biological systems. There is growing evidence that the phenomenon of "stochastic resonance", (SR) [7] may play a role in the extreme sensitivity exhibited by various sensory neurons: the cricket cercal system [8], human tactile sensation [9], and hair mechanoreceptors in the tail fan of the crayfish [10]. However, these processes all occur at the periphery of the nervous system, and it is interesting to consider whether similar phenomena may occur within the brain. In fact, SR has been shown to occur in a hippocampal slice in vitro [11] but it is not clear whether this effect serves any purpose or is merely an artifact.

In this paper we examine and analyze the dynamics of the periodically driven noisy Haken model. We show that it undergoes a resonant type behavior which is reminiscent of stochastic resonance, and suggest a biological interpretation of our results.

\section{HAKEN'S COMPETITIVE NETWORK}

Consider a single-layer network of $N$ neurons and denote the state of the $n$th neuron by $q_{n} \in \mathrm{R}$ with $n=1, \ldots, N$. In
Haken's original model the network evolves according to the gradient dynamics [3]

$$
\dot{q}_{n}=-\frac{\partial V(\mathbf{q})}{\partial q_{n}}=\left[1-2 \Delta(\mathbf{q})+q_{n}^{2}\right] q_{n},
$$

with

$$
V(\mathbf{q})=-\frac{1}{2} \Delta(\mathbf{q})+\frac{1}{2} \Delta(\mathbf{q})^{2}-\frac{1}{4} \sum_{n} q_{n}^{4}
$$

and

$$
\Delta(\mathbf{q})=\sum_{n} q_{n}^{2}
$$

represents a form of global coupling. Note that the lack of local interactions implies that there is no natural network topology. Figure 1 shows a plot of the potential $V(\mathbf{q})$ for a network of two neurons, $q_{1}$ and $q_{2}$.

Equation (1) is invariant under the transformation $\mathbf{q} \rightarrow$ -q. Moreover, $q_{n}(t) \geqslant 0$ for all $t>0$ and $n$ if $q_{n}(0) \geqslant 0$ for all $n$. For suppose that $q_{n}(t)=0$ and $q_{m}(t) \geqslant 0$ for all $m$ $\neq n$. Setting $q_{n}=0$ on the right-hand side of Eq. (1) shows that $\dot{q}_{n}(t) \geqslant 0$. That is, $q_{n}$ cannot cross over to the negative real axis. The network converges to one of the stationary states of the potential $V$, that is,

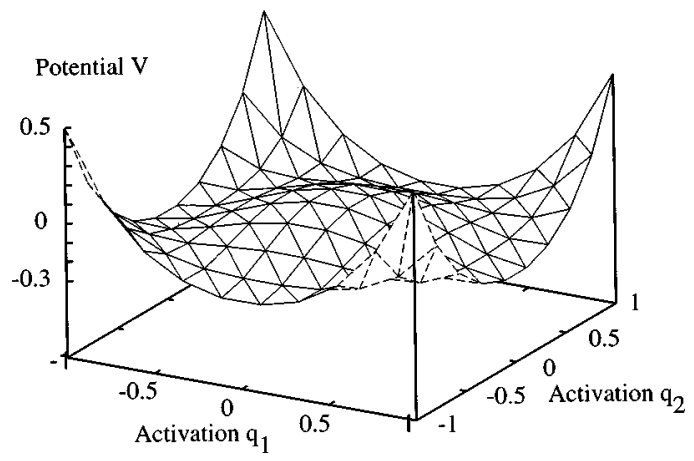

FIG. 1. Plot of the potential for the two neuron nondiffusive network. Minima are shown at $(1,0),(0,1),(-1,0),(0,-1)$ and a maximum at $(0,0)$. All quantities are in dimensionless units. 


$$
-\frac{\partial V}{\partial q_{n}}=q_{n}^{3}+(1-2 \Delta) q_{n}=0
$$

for all $n$. Thus the equilibria of Eq. (1), which we denote by $\overline{\mathbf{q}}$, satisfy $\bar{q}_{n}=0$ or $\bar{q}_{n}=\sqrt{2 \Delta-1}$ with $\Delta$ determined selfconsistently. Hence the set of stationary states can be divided into $N+1$ classes, each of which is determined by the number $p$ of excited sites. For a given $p, \Delta=p /(2 p-1)$ and the corresponding potential at a stationary state is

$$
V^{(p)}=-\frac{p}{(8 p-4)} .
$$

Linear stability analysis $[3,6]$ establishes that only the stationary states $p=1$ are stable, whereas all other stationary states are either unstable $(p=0)$ or saddle points $(p>1)$. For each state in the class $p=1$, there exists a single excited site, $n_{0}$ say, such that $\bar{q}_{n}=\delta_{n_{0}, n}$. Moreover $\Delta=1$ and $V^{(1)}$ $=-1 / 4$. These are the $N$ strictly localized ground states of the network. There are two homogeneous stationary states given by the vacuum state $p=0$ and the dissipative state $p$ $=N$. The former satisfies $q_{n}=0$ for all $n$ and $V^{(0)}=0$ and the latter has

$$
\bar{q}_{n}=\frac{1}{\sqrt{2 N-1}} \forall n
$$

and

$$
V^{(N)}=-\frac{N}{(8 N-4)} .
$$

In the large $N$ limit the dissipative state becomes pointwise identical to the vacuum state but has lower energy, $V^{(\infty)}=$ $-1 / 8$. Also note that for an infinite lattice the dissipative state is marginally stable.

We conclude that the ground states of the system consist of strictly localized states in which only one site is excited and the remainder quiescent; the particular ground state selected depends on the initial data and/or additional applied inputs. If there are no external inputs, then the excited neuron is the one with the highest initial activity. In other words, the network dynamically realizes a winner-takes-all strategy. Such networks are typically termed competitive networks. Competitive networks signify their outputs by the firing of a single neuron, or a small proximal group of neurons. They thus classify data by the firing of the same neuron(s) for all inputs that belong to a single category. Electrophysiological recordings from single cortical cells indicate that in the brain the representation of sensory information is not encoded by the global activity of the entire cortex, but rather by the firing patterns of small groups of neurons (see Ref. [12], and references therein). Competitive networks therefore provide rudimentary models of how perception and categorization occur in real brains [13]. It can also be shown that such networks are equivalent to associative memories [14], and Haken has demonstrated that this particular network can perform associative recall of digitized photographs [3].

One obvious drawback to this model is its inability to learn. Output states are "hard wired" into the dynamics, and all have the same size basins of attraction. For a truly biological system one would want the facility to learn new categories, and also to emphasize or deemphasize others. In fact, Haken's original formulation includes variable synaptic strengths but for our purposes we set them all to be equal to unity and thus neglect their effect.

\section{THE DIFFUSIVE HAKEN MODEL}

It is clear that competitive networks with single output neurons are not robust to degradation: if a single neuron is destroyed then the entire corresponding category is lost. Recently, one of us [5] has shown that the inclusion of a diffusive term in the potential of the original Haken model can delocalize the ground states. For certain values of the coupling strength there can exist a balance between the effects of the diffusion and of the localizing potential, which yields new states that are localized excitations (or bubbles) distributed over many neurons. These "bubbles" represent a very robust coding of information since neighboring cells aid the reconstruction of lost information following the "death" of a single cell. Furthermore, Kohonen [15] has shown that such "bubbles" enable the construction of topographic maps.

We now impose a $d$-dimensional square lattice topology on the network; the diffusive Haken model has a potential

$$
U(q)=\frac{\alpha}{2} \sum_{\langle m, n\rangle}\left(q_{m}-q_{n}\right)^{2}+V(\mathbf{q}),
$$

where $\langle m, n\rangle$ denotes summation over nearest neighbor pairs. The first term on the right-hand side of Eq. (8) represents a diffusive interaction with coupling strength $\alpha$. Using the idea of an anticontinuum limit [16] a uniform continuation from the zero diffusive coupling $(\alpha=0)$ case can be performed. We denote the state of the network by $Q(\alpha, \Delta)$. Stationary states satisfy

$$
(1-2 \Delta) q_{n}+q_{n}^{3}+\alpha \sum_{\langle m, n\rangle}\left(q_{m}-q_{n}\right) \equiv G(Q, \alpha, \Delta)=0 .
$$

For a given $\Delta \equiv \Delta_{0}, \Delta_{0}>1 / 2$, and $\alpha=0$ the equilibria of Eq. (9) satisfy $\bar{q}_{n}=0$ or $\bar{q}_{n}= \pm \sqrt{2 \Delta_{0}-1}$ (if negative solutions are included). Denote the Jacobian $\partial G / \partial Q$ by $\delta G$. Since $\left[\delta G\left(\bar{Q}, 0, \Delta_{0}\right)\right]_{n m}=\delta_{n, m} \lambda_{n}$ with $\lambda_{n}=-\left(2 \Delta_{0}-1\right)$ if $\bar{q}_{n}=0$ and $\lambda_{n}=2\left(2 \Delta_{0}-1\right)$ if $\bar{q}_{n} \neq 0, \delta G$ is invertible at the stationary point $(\bar{Q}, 0, \Delta)$. Hence one can use the implicit function theorem to show that for sufficiently small coupling $\alpha$ there exist local continuations of each $\bar{Q}$ for sufficiently small $\alpha$ [5]. Furthermore one can show that to a first approximation each state has a potential

$$
U_{\min }(\alpha) \approx V_{\min }+d \alpha
$$

In fact, such states persist for all values of $\alpha$ in one dimension, whereas for $d>1$ there exists a critical coupling $\alpha_{c}(d)$ beyond which localized ground states cease to exist and the effects of diffusion dominate. The critical coupling can also be computed [5] and is found to be 


$$
\alpha_{c}(d)=\frac{1}{10 d}
$$

The analysis of Ref. [5] holds for more general couplings than just a diffusive one, the only criteria being that the coupling strength decays exponentially with distance.

\section{STOCHASTIC DYNAMICS OF THE NONDIFFUSIVE MODEL}

We select two neurons $n=1,2$, say, and drive the network with the weak periodic bias

$$
I_{n}(t)=\varepsilon\left[\delta_{n, 1} \cos ^{2}(\Omega t)+\delta_{n, 2} \sin ^{2}(\Omega t)\right] .
$$

We further impose $\varepsilon$ small, so that the bias itself is unable to cause transitions between states, i.e., we have a subthreshold forcing. We also introduce a local additive Gaussian noise for each neuron. This corresponds to an internal noise of, say, thermal origin. Equation (1) then becomes

$$
\dot{q}_{n}(t)=-\frac{\partial V(\mathbf{q})}{\partial q_{n}}+\sigma \xi_{n}(t)+I_{n}(t),
$$

where $\xi_{n}(t)$ is a zero-mean Gaussian white noise process with $\left\langle\xi_{n}(t)\right\rangle=0$ and $\left\langle\xi_{m}\left(t_{1}\right) \xi_{n}\left(t_{2}\right)\right\rangle=\delta_{m, n} \delta\left(t_{1}-t_{2}\right)$. Thus the noise is uncorrelated between neurons, and has variance $D=\sigma^{2}$.

Numerical simulations show that at low noise levels the network tends to remain in one of two possible output states, and that switching events between these two states occur exceedingly rarely. In the limit of zero noise these states correspond to $q_{n}=\delta_{n, 1}$ and $q_{n}=\delta_{n, 2}$, and the occupied one depends on initial conditions. As the noise is gradually increased the network begins to jump between output states with a transition rate that is partly entrained with the driving force. For high noise levels the network randomly flips between output states and there is no synchrony with the driving signal. Simulations also show that in the entrained regime there is a clear separation of time scales for the system, the two scales are the time to relax to an output state $t_{\text {relax }}$ and the mean residence time of an output state, $t_{\text {res }}$, with $t_{\text {res }} \gg t_{\text {relax }}$. We therefore make the adiabatic assumption and neglect the relaxation time.

To quantify the behavior of the network we tabulate and histogram the residence times of an output state (which is equivalent to the transition time out of the state). Depending on the level of noise, the resulting distribution typically displays peaks centered at

$$
T_{p}=\left(p-\frac{1}{2}\right) T_{0}, \quad p \in \mathbb{Z}
$$

where $T_{0}=\pi / \Omega$ is the driving period. These peaks are superimposed on an exponentially decaying background (see inset to Fig. 2). We denote the height of the $k$ th peak by $h_{k}$. Each $h_{k}$ passes through a maximum as a function of both noise strength and the forcing period [17]. In Ref. [17] it was suggested that for a particular driving frequency $\nu=T_{0}^{-1}$, stochastic resonance is attained at the particular noise strength $\sigma$ for which the height of the first harmonic, $h_{1}$, is

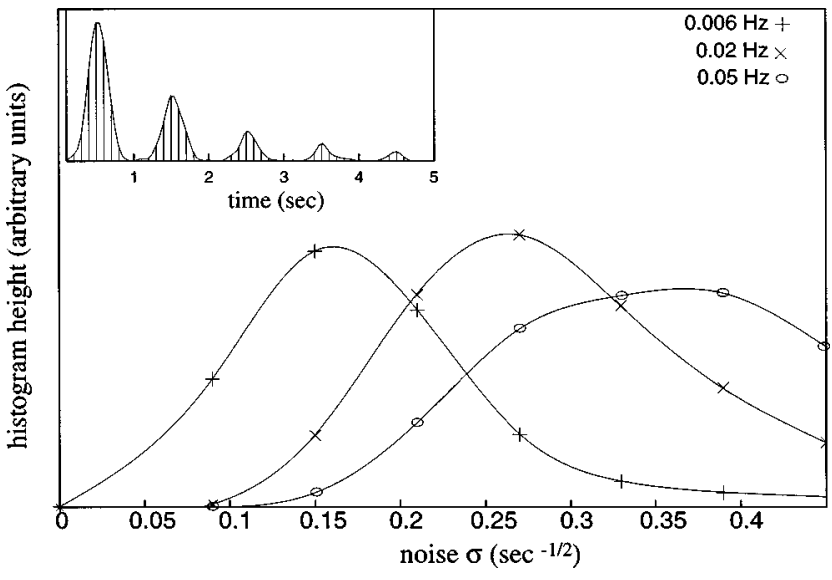

FIG. 2. Variation of $h_{1}$ with noise strength $\sigma$ for the nondiffusive network. Three different values of the driving frequency $\nu$ are shown, and as $\nu$ increases $\sigma_{\text {opt }}$ increases. The inset shows a typical exponentially decreasing histogram of residence times: time is in multiples of $T_{0}$ (see text).

maximized. However, the connection between Gammaitoni et al.'s definition and a maximum in the power spectral density of the system, which is the signature of stochastic resonance, has yet to be established. In fact this loose end is presently the subject of some controversy, and for this reason we hesitate to describe this effect as stochastic resonance, but instead name it a "resonance in the sense of Gammaitoni et al." With this caveat, Fig. 2 shows how $h_{1}$ for the Haken network varies with noise strength $\sigma$. Several values of the driving frequency are shown. It is seen that the maximal value of $h_{1}$ occurs at a nonzero value of $\nu$, and that as $\nu$ increases, this maximum is shifted to higher noise levels. Noise assisted synchronization and a dependence of the optimal noise strength on the driving frequency are features shared with systems displaying stochastic resonance.

It is not a priori obvious that we can reduce the effective dimensionality of our system. However, for low noise levels $q_{n}(t) \approx 0 \forall n \neq 1,2$, and thus to a good approximation Eq. (13) reduces to a two-dimensional system $(n=1,2)$ with $V$ given by Eq. (1) for $N=2$. In the positive quadrant the potential $V\left(q_{1}, q_{2}\right)$ has two minima $\mathbf{q}_{a}$ at $(1,0)$ and $(0,1)$, and a saddle at $\mathbf{q}_{s}=(1 / \sqrt{3}, 1 / \sqrt{3})$ with $V\left(\mathbf{q}_{s}\right)=-1 / 6$, see Fig. 1 . Since the periodic forcing is chosen to be positive valued the system is retained in the positive quadrant and thus one can neglect the effects of the minima at $(-1,0)$ and $(0,-1)$. Recall that the system remains in the positive quadrant in the absence of any noise or external forcing. We may therefore reduce $V$ to a two-dimensional bistable potential, provided that $\epsilon$ is not too small and the noise is not too large.

To qualify as a resonance phenomenon, some internal frequency of the system must match the driving frequency. In Ref. [17] it was proposed that resonance takes place when the driving frequency $\nu$ matches, or resonates with the mean transition rate due to noise of the unforced system. It was further suggested that this occurs when $h_{1}$ is maximal. In fact, when $h_{1}$ is maximal very few transitions occur at other harmonics, and thus the first harmonic dominates the histogram. Under these conditions, the mean first passage time, which is equal to the first moment of the histogram, is close to the driving period. Thus we justify naming this effect a resonance. 


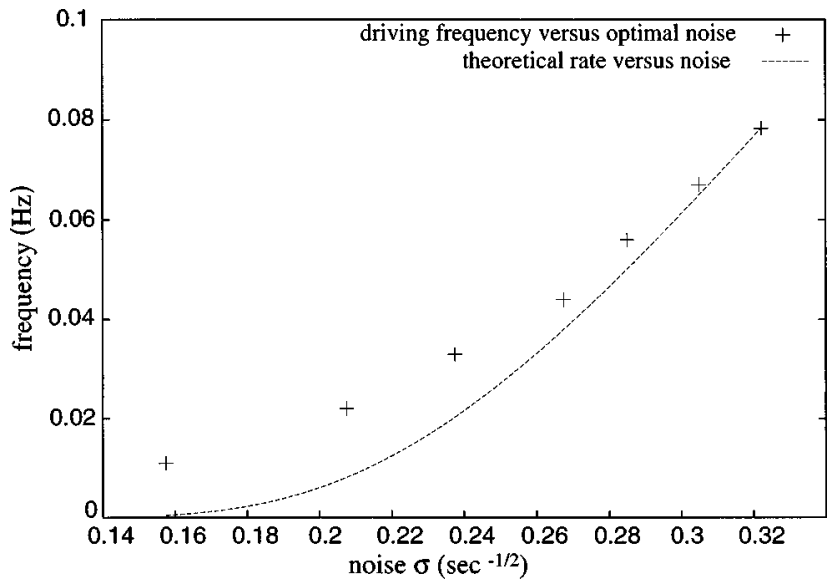

FIG. 3. The matching of the theoretical escape rate $r$ with twice the driving frequency $\nu$.

We assume that all transition events occur at the saddle point, reasoning that transitions at all other points are exponentially less likely. It can therefore be shown that the mean escape rate from a given minimum in an unperturbed multidimensional multistable potential is given by the Kramers rate formula [18]

$$
r(\sigma)=\frac{\lambda}{2 \pi} \sqrt{\frac{\operatorname{det} \mathbf{H}\left(\mathbf{q}_{a}\right)}{\left|\operatorname{det} \mathbf{H}\left(\mathbf{q}_{s}\right)\right|}} \exp \left(\frac{-2 \delta V}{\sigma^{2}}\right)
$$

The Hessian $\mathbf{H}$ of the potential has components

$$
H_{m n}=\frac{\partial V^{2}}{\partial q_{m} \partial q_{n}}
$$

and is evaluated at the minima, $\mathbf{q}_{a}$, and the saddle, $\mathbf{q}_{s} . \lambda$ is the positive eigenvalue of the Hessian of the potential at the saddle, and $\delta V=V_{s}-V_{a}=1 / 12$ is the height of the potential barrier at the saddle. For our system the prefactor in Eq. (15) has the value 0.39 .

Resonance occurs when the time for the system's mean residence in one minimum is close to half the driving period [17]. This is equivalent to the condition $r=2 \nu$. Therefore, given a driving frequency $\nu$ we may experimentally ascertain $\sigma_{\text {opt }}$, the optimal noise level for resonance in the system. Using Eq. (15), $\sigma_{\text {opt }}$ determines $r_{\text {opt }}$, the corresponding theoretical escape rate. Thus we may compare $r_{\text {opt }}$ with the original driving frequency $\nu$. Figure 3 shows plots of $r(\sigma)$ versus noise strength $\sigma$, and $2 \nu$ versus optimal noise strength $\sigma_{\mathrm{opt}}$. It is seen that the optimal noise level matches well that predicted by the theory.

Note that in the absence of any periodic forcing the histogram of residence times is essentially a decaying exponential as predicted from Kramers' theory, which is in contrast to the multimodal structure shown in Fig. 2. If one determines the variation in the height of the histogram at a residence time equal to one of the periods of the forced case, one also sees the histogram height pass through a maximum. However, this occurs at much lower noise levels (i.e., there is no matching of time scales) and is simply an artifact of the sampling.
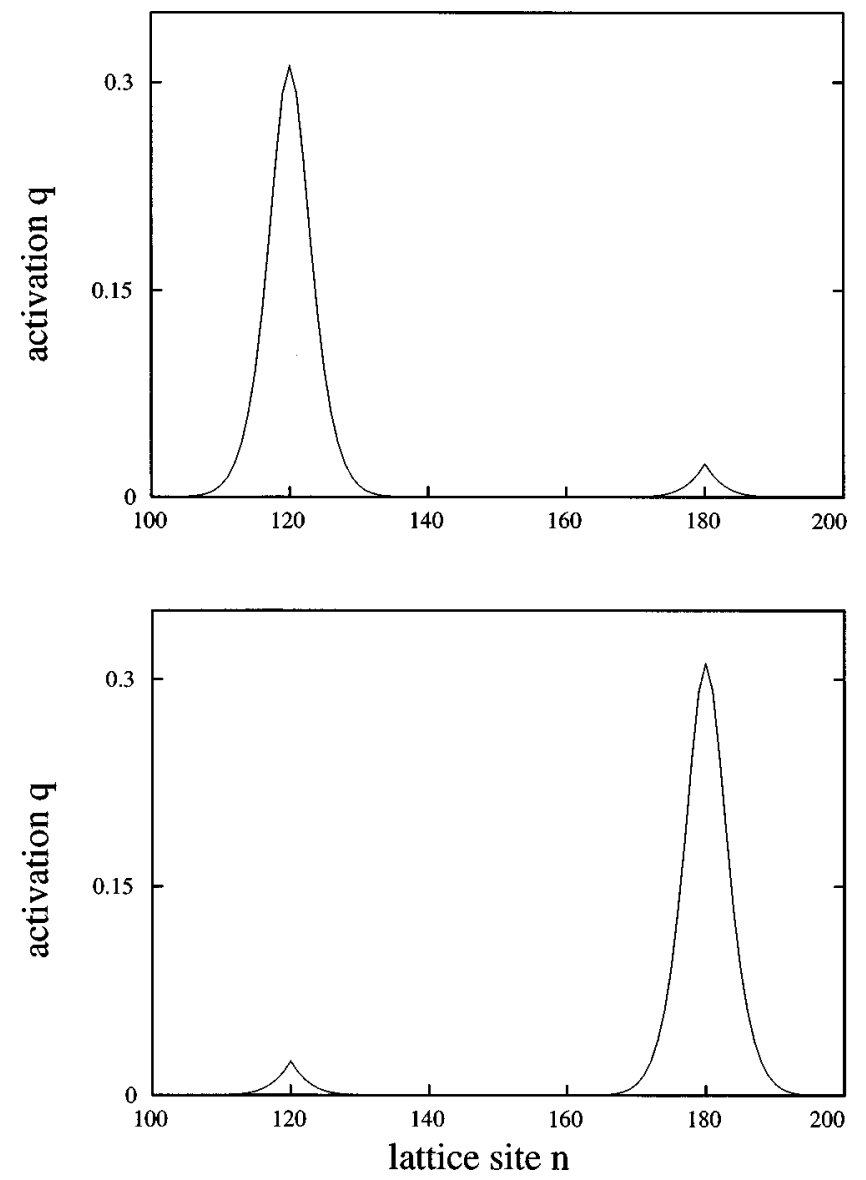

FIG. 4. Two snapshots of the evolution of the network with diffusive coupling $\alpha=0.5$ : before and after the network has flipped between states. For clarity the low noise case is shown.

\section{STOCHASTIC DYNAMICS OF THE DIFFUSIVE MODEL}

For concreteness, consider a one-dimensional lattice and select two neurons separated by $r$ lattice sites with $r$ sufficiently large such that the ground states centered at the two sites have very little overlap. As in the nondiffusive case we introduce local additive noise together with a periodic stimulation of the two selected neurons given by $\varepsilon \cos ^{2}(\Omega t)$ and $\varepsilon \sin ^{2}(\Omega t)$, respectively. The equations of motion are thus of the form

$$
\dot{q}_{n}(t)=-\frac{\partial U(\mathbf{q})}{\partial q_{n}}+\sigma \xi_{n}(t)+I_{n}(t),
$$

with $U(\mathbf{q})$ given by Eq. (8). A resonant effect is again observed with the system switching between the states localized about the two centers (see Fig. 4).

Plots of $h_{1}$ versus $\sigma$ are shown in Fig. 5 for various coupling strengths. We observe that as $\alpha$ is increased, the maximum of $h_{1}$, corresponding to resonance, is shifted to lower noise levels. This may be explained by noting that increasing $\alpha$ causes a decrease in the barrier height $\delta U(\alpha)$ and thus an increase in the unperturbed transition rate (15). We indicate below a method for calculating $\delta U(\alpha)$.

When $\alpha$ is sufficiently large, the localized solutions of the one-dimensional diffusive Haken model (in the absence of noise and external forcing) are distributed over many lattice 


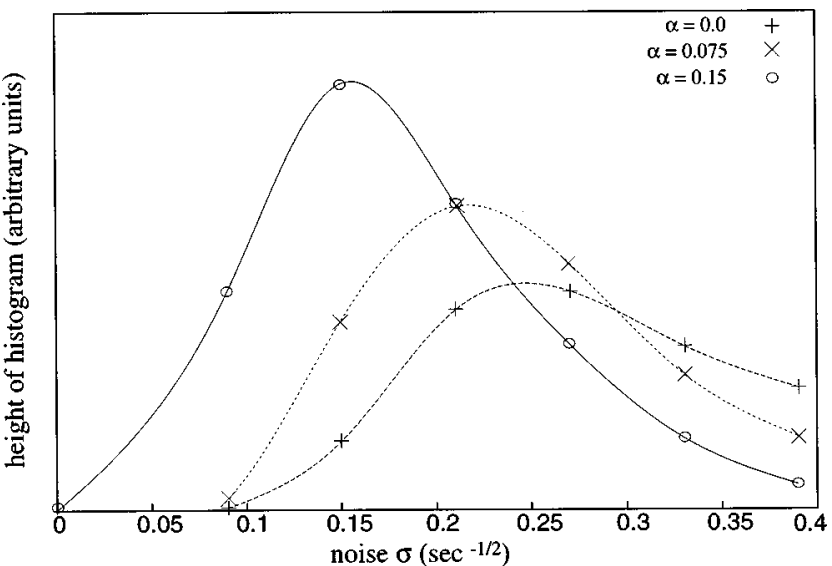

FIG. 5. Variation of $h_{1}$ with noise strength $\sigma$ for the diffusive network. Three different values of the coupling strength $\alpha$ are shown, and as $\alpha$ increases $\sigma_{\mathrm{opt}}$ decreases. The driving frequency $\nu$ is held constant.

sites suggesting that they can be approximated by a continuum version of the model. The potential governing the gradient dynamics of the continuum model takes the form

$$
U[q, \alpha]=\int_{-\infty}^{\infty} d x\left[\frac{\alpha}{2}\left(\frac{\partial q(x)}{\partial x}\right)^{2}-\frac{q(x)^{4}}{4}\right]-\frac{\Delta}{2}+\frac{1}{2} \Delta^{2},
$$

with

$$
\Delta[\mathbf{q}]=\int_{\mathrm{R}} d x q(x)^{2}
$$

and $\alpha$ a renormalized diffusion constant. By means of the Euler-Lagrange equation, stationary solutions of the dynamics satisfy

$$
\alpha \frac{d^{2} q}{d x^{2}}=(2 \Delta[\mathbf{q}]-1) q(x)-q(x)^{3} .
$$

Localized states can now be interpreted as finite energy configurations or instantons of the continuum model. Using phase-plane analysis it can be shown that for fixed $\Delta$ one has the following analytical expression for an instanton (centered at $x=0)[4,6]$ :

$$
q(x)=q_{0}\left[\cosh \left(\frac{q_{0} x}{\sqrt{2 \alpha}}\right)\right]^{-1} .
$$

The amplitude of the instanton is

$$
q_{0}=\sqrt{(2 \Delta-1) 2} .
$$

This leads to a self-consistency condition for $\Delta$ of the form

$$
\Delta=4 \sqrt{\alpha(2 \Delta-1)},
$$

which has real solutions provided that

$$
\alpha \geqslant \bar{\alpha}=1 / 16 \text {. }
$$

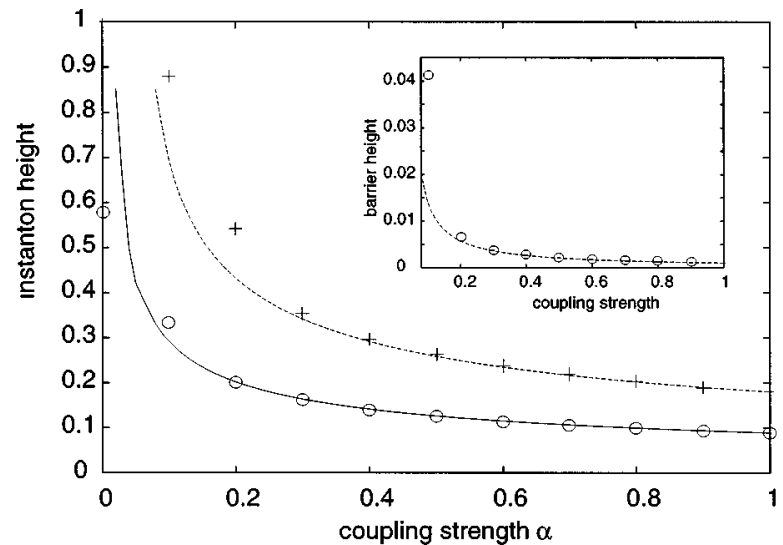

FIG. 6. The variation of the instanton amplitudes, $q_{o}$ and $q_{0}^{\prime}$, with coupling strength $\alpha$. The dashed curve shows the singlet amplitude [Eq. (25)], the bold one shows the doublet amplitude [Eq. (28)]. Simulation results are also shown: results for the singlet state are represented by a plus sign, and for the doublet state by a circle. Inset: The variation of the barrier height with coupling strength $\alpha$ is shown both analytically (solid line) and numerically (circles).

Keeping only the lower energy solution, the amplitude of the instanton as a function of the coupling $\alpha$ is

$$
q_{0}(\alpha)=\sqrt{2 \frac{\alpha}{\bar{\alpha}}}\left(1-\sqrt{1-\frac{\bar{\alpha}}{\alpha}}\right) .
$$

It follows that the energy of the instanton is [note that Eq. (10) is only valid in the limit $\alpha \rightarrow 0$ ]

$$
U_{\min }(\alpha)=F\left(q_{0}(\alpha), \alpha\right),
$$

where

$$
F\left(q_{0}, \alpha\right)=-\sqrt{2 \alpha} q_{0}+4 \alpha q_{0}^{2}-\sqrt{2 \alpha} \frac{q_{0}^{3}}{6} .
$$

We can now determine the barrier height for transitions between two single-instanton states centered at different sites $x_{1}$ and $x_{2}$ (cf. Fig. 4) under the assumptions that $x_{1}, x_{2}$ are well separated on the lattice and that the most probable path of escape is via a saddle consisting of an instanton doublet centered about the two selected sites. Solving the selfconsistency condition for $\Delta$ we find that the height of each instanton in the doublet is

$$
q_{0}^{\prime}(\alpha)=\sqrt{2 \frac{\alpha}{\bar{\alpha}}}\left(2-\sqrt{4-\frac{\bar{\alpha}}{\alpha}}\right), \text { with } q_{0}^{\prime}<q_{0} .
$$

Figure 6 shows how the amplitudes of the single instanton, and a member of the doublet, vary with the coupling strength $\alpha$; as expected, the continuum limit becomes invalid for small $\alpha$.

The energy of the doublet (assuming that the local interaction energy of the two instantons can be neglected) is

$$
U_{d}(\alpha)=2 F\left(q_{0}^{\prime}(\alpha), \alpha\right)+8 \alpha q_{0}^{\prime}(\alpha)^{2}
$$

and the required barrier height is 


$$
\delta U(\alpha)=U_{d}(\alpha)-U_{\min }(\alpha) .
$$

The inset to Fig. 6 demonstrates that the barrier height rapidly decreases with increasing coupling strength, as we expect. One consequence of this is that there appears to be a tradeoff between the strength of the local coupling, $\alpha$, and hence the system's ability to withstand damage, and the robustness of the system to noise. Strong coupling $(\alpha>0.2)$ means that information about an output state is dispersed over many neurons, making a resilient system. However, this also means that the barrier height between output states becomes so small that noise induced transitions become important, even for low noise levels. Thus the network is unable to function as a classifier.

\section{CONCLUSION}

We have shown that a competitive neural network model with weak noise and a subthreshold driving signal can exhibit a resonant behavior that is akin to stochastic resonance. Stochastic resonance is a phenomenon whereby random fluctuations and noise can enhance the detectability and/or the coherence of a weak signal in certain nonlinear dynamical systems (see, e.g., Ref. [7], and references therein). We have also seen that this resonance persists when diffusive coupling is introduced into the model: a feature which induces stability and robustness into the network. We believe that Haken's model provides a useful model for classification tasks in the same way that the Hopfield model, though biologically implausible, is a viable metaphor for associative memory.

Recalling that a main impetus for investigating competitive networks is to model mental categorization tasks, we look for commensurate resonant behavior in biological systems. Recent psychophysical evidence suggests that stochastic resonance may feature in high level brain function: Simo- notto et al. [19] have shown that SR may be exploited during the recognition of static visual images that have been distorted by time varying noise. Chialvo and Apkarian [20] and Riani and Simonotto [21] report on psychophysical experiments where SR is observed in the perceptual transitions between either facet of an ambiguous figure, such as the Necker cube. In both Ref. [20] and Ref. [21] the image, or ambiguous figure, is given a weak periodic transformation corresponding to a weak signal but the noise has different origins. If we ascribe a ground state of Haken's model to each possible interpretation of the ambiguous figure, then our treatment is suggestive of these results. Riani and Simonotto [22] use the alternative formulation of a constraint satisfaction network, which is equivalent to a suitably configured Hopfield network, to model their experimental results. Such a formulation was first introduced by Rumelhart et al. within the context of connectionist models [23], but was then extended by Riani and Simonotto to demonstrate stochastic resonance. Connectionist models describe psychological phenomena in terms of such mental processes as ideas and schemata, and are thus high level explanations: in the model of Rumelhart et al. each node of the network corresponds to a possible hypothesis about the network's input, rather than an individual neuron. In contrast, competitive networks attempt to provide a low level description of neural activity, and therefore such models may help to explain how a neural architecture can subsume mental activity.

Despite superficial similarities, the behavior of our model must be distinguished from " array enhanced stochastic resonance", [24] which is a noise induced phase locking phenomenon. Our analysis has shown that for small diffusive coupling the stochastic Haken model can be approximated by a bistable system and thus has more in common with traditional SR models.
[1] N. V. Swindale, Network 7, 161 (1996).

[2] J. H. Kaas, R. J. Nelson, M. Sur, C. S. Lin, and M. M. Merzenich, Science 204, 521 (1979).

[3] H. Haken, Synergetic Computers and Cognition (SpringerVerlag, Berlin, 1991).

[4] M. Schmutz and W. Banzhaf, Phys. Rev. A 45, 4132 (1992).

[5] P. C. Bressloff, Phys. Rev. Lett. 75, 962 (1995).

[6] P. C. Bressloff, Physica D 110, 195 (1997).

[7] F. Moss, D. Pierson, and D. O'Gorman, Int. J. Bifurcation Chaos Appl. Sci. Eng. 4, 1383 (1994); K. Wiesenfeld and F. Moss, Nature (London) 373, 33 (1995); A. R. Bulsara and L. Gammaitoni, Phys. Today 49 (3), 39 (1996).

[8] J. E. Levin and J. P. Miller, Nature (London) 380, 165 (1996).

[9] J. J. Collins, T. T. Imhoff, and P. Grigg, Nature (London) 383, 770 (1996).

[10] J. K. Douglas, L. Wilkens, E. Pantazelou, and F. Moss, Nature (London) 365, 337 (1993).

[11] B. J. Gluckman, T. I. Netoff, E. J. Neel, W. L. Ditto, M. L. Spano, and S. J. Schiff, Phys. Rev. Lett. 77, 4098 (1996).

[12] P. Földiák and M. P. Young, The Handbook of Brain Theory and Neural Networks, edited by M. Arbib (MIT Press, Cambridge, MA, 1995).

[13] C. von der Malsburg, Kybernetik 14, 85 (1973).

[14] T. Kohonen, Self Organization and Associative Memory (Springer-Verlag, Berlin, 1984).

[15] T. Kohonen, Biol. Cybern. 43, 59 (1982).

[16] S. Aubry, Physica D 86, 284 (1995).

[17] L. Gammaitoni, F. Marchesoni, and S. Santucci, Phys. Rev. Lett. 74, 1052 (1995).

[18] P. Hänggi, P. Talkner, and M. Borkovec, Rev. Mod. Phys. 62, 251 (1990).

[19] E. Simonotto, M. Riani, C. Seife, M. Roberts, J. Twitty, and F. Moss, Phys. Rev. Lett. 78, 1186 (1997).

[20] D. R. Chialvo and A. Apkarian, J. Stat. Phys. 70, 375 (1993).

[21] M. Riani and E. Simonotto, Nuovo Cimento D 17, 903 (1995).

[22] M. Riani and E. Simonotto, Phys. Rev. Lett. 72, 3120 (1994).

[23] D. E. Rumelhart, P. Smolensky, J. L. McClelland, and G. E. Hinton, Parallel Distributed Processing (MIT Press, Cambridge, MA, 1986), Vol. 2, Chap. 14.

[24] J. F. Lindner, B. K. Meadows, W. L. Ditto, M. E. Inchiosa, and A. R. Bulsara, Phys. Rev. Lett. 75, 3 (1995). 